\title{
Clastogenicity of Beta-Caryophyllene in Mouse
}

\author{
Dolores Molina-Jasso, Isela Álvarez-GonzÁlez, and Eduardo Madrigal-Bujaidar* \\ Laboratorio de Genética, Escuela Nacional de Ciencias Biológicas, Instituto Politécnico Nacional; Carpio y Plan de \\ Ayala, Col. Santo Tomas. México, D. F. CP. 11340, México. \\ Received October 3, 2008; accepted December 4, 2008; published online December 11, 2008
}

\begin{abstract}
Beta-caryophyllene (BC) is a chemical found in the essential oil of numerous plants which have shown various biomedical properties. In the present investigation we examined for the first time its genotoxic capacity in vivo by evaluating its potential to induce micronuclei in mouse. In a first assay we administered three doses of the compound once, and in a second assay, we administered three doses of $\mathrm{BC}$ in three consecutive days. In each assay, the results were compared with those obtained in a group of mice administered corn oil and in another treated with adryamicin. Our results revealed no genotoxic effect by $\mathrm{BC}$ in the two assays. Moreover, no bone marrow cyotoxicity was observed when the proportion of polychromatic erythrocytes was determined with respect to the number of normochromatic erythrocytes. Therefore, our study showed the relevance of extending research on $\mathrm{BC}$ regarding its beneficial properties.
\end{abstract}

Key words beta-caryophyllene; micronucleus; mouse

Plants are used for various purposes including their cosmetic, nutritive, and biomedical properties. In this context, a number of therapeutic effects have been determined for different plant extracts or specific compounds. Beta-caryophyllene (BC) in particular, is a volatile sesquiterpene which has been detected in the essential oil of plants such as Salvia, Artemisia and Eugenia, as well as in various spices which include clove, origanum, thyme, pepper and cinnamon. ${ }^{1-3)}$ The compound participates in the odor and flavor of the plants, besides, due to its fragrance, BC is used in creams, lotions, and soaps. ${ }^{4)}$ On the other hand, an anti-anemic effect was suggested for BC in the middle of the last century, and more recently, studies in mouse, rat, rabbit, bacteria, and mammalian cell lines have shown its effect as an anti-inflammatory agent and antimutagen, a protector of gastric damage and oxidative stress, a local anesthetic, a potential medication against acne, and as an anticarcinogen due to its capacity to detoxify xenobiotics or to attack cancerous cell lines. ${ }^{1,2,5)}$

However, in spite of the potentially useful biomedical properties mentioned above, only one report concerning the effect of $\mathrm{BC}$ on DNA has been published to date. The authors evaluated its mutagenic potential by using the bacterial reverse mutation assay and obtained negative results ${ }^{2}$; therefore, the study of its genotoxic potential in an in vivo assay was necessary. In this investigation we determined the effect of $\mathrm{BC}$ on the induction of micronucleated polychromatic erythrocytes (MNPE) in mouse, as well as its cytotoxicity by detecting its capacity to modify the bone marrow polychromatic erythrocyte rate in the same model.

\section{MATERIAL AND METHODS}

Chemicals and Animals Beta-caryophyllene (97\% pure), acridine orange corn oil and adriamycin (AD) (98\% pure) were obtained from Sigma Chemicals (St. Louis, MO, U.S.A.), and dibasic sodium phosphate, monobasic sodium phosphate, methanol and ethanol were acquired from J. T. Baker (Mexico City).

For the experiment we used six-week old male mice (strain $\mathrm{NIH}$ ) weighing $25 \mathrm{~g}$ obtained from the National Institute of Hygiene (Mexico City). The animals were kept in polypropy- lene cages at $23^{\circ} \mathrm{C}$ in a $12 \mathrm{~h}$ dark-light schedule, and allowed to freely consume food (Rodent Laboratory chow 5001, Purina) (Mexico City) and water. The experimental protocol was approved by the Committee of Ethics and Biosecurity of the National School of Biological Sciences.

Determination of $\mathbf{L D}_{\mathbf{5 0}}$ The acute toxicity of $\mathrm{BC}$ was determined according to the method described by Lorke. ${ }^{\text {) }}$ The compound was dissolved in corn oil and administered orally to mice. In a first step, the animals received doses of 10,100 , and $1000 \mathrm{mg} / \mathrm{kg}$. We detected no mortality or other signs of toxicity along $7 \mathrm{~d}$ post-administration. Therefore, we administered up to $5000 \mathrm{mg} / \mathrm{kg}$ of $\mathrm{BC}$ and again, no sign of toxicity was observed in the tested animals.

Genotoxicity Protocols In the first assay we organized five groups with five individuals each as follows: a control group administered orally with $0.1 \mathrm{ml} / \mathrm{g}$ of corn oil; three groups treated orally with BC in doses of 20, 200, and $2000 \mathrm{mg} / \mathrm{kg}$, respectively; and a positive control group injected intraperitoneally with $\mathrm{AD}(2.5 \mathrm{mg} / \mathrm{kg})$. Blood samples from the tail of each mouse were obtained before the chemical administration and at 24, 48, 72, and $96 \mathrm{~h}$ post-administration.

A second experimental protocol was performed to confirm the results obtained in the first assay. The mice were organized and treated as indicated above except for the dose of AD $(1.0 \mathrm{mg} / \mathrm{kg})$. As for the other groups we used $0.1 \mathrm{ml} / \mathrm{g}$ of corn oil, and 20,200 , and $2000 \mathrm{mg} / \mathrm{kg}$ of the tested chemical administered to three groups. The main difference with the previous protocol was that the administration of chemicals to the corresponding groups was made in three consecutive days. Blood samples from the tail of each mouse were also taken before the chemical administration and at 24, 48, 72, and $96 \mathrm{~h}$ post-administration.

Slide Preparation, Staining, and Micronuclei Determination In both protocols, two blood smears per mouse were made, fixed in methanol for $5 \mathrm{~min}$ and stained for $1 \mathrm{~min}$ with a $0.1 \%$ solution of acridine orange made in phosphate buffered saline (PBS), $\mathrm{pH}$ 6.8. We then rinsed the slides three times with PBS and observed them in a fluorescence microscope (Carl Zeiss, Axioskop) with a $420 \mathrm{~nm}$ filter. Under these conditions, micronuclei show green-yellowish fluores- 
cence, polychromatic erythrocytes (PE) have an orange color, and normochromatic erythrocytes (NE) show very low fluorescence. ${ }^{7)}$ The capacity of $\mathrm{BC}$ to induce micronuclei was determined in $1000 \mathrm{PE}$ per mouse in each of the five time points of the assays. Besides, we took advantage of our test model to determine the effect of the chemical on bone marrow cytotoxicity, by quantifying (in 1000 erythrocytes per mouse) the proportion of PE with respect to the number of normochromatic erythrocytes (PE/NE percent). The obtained results were statistically evaluated with the ANOVA and the Student $t$ tests.

\section{RESULTS AND DISCUSSION}

The results on the rate of MNPE obtained in the first assay are shown in Table 1. We found the number of micronuclei induced by $\mathrm{BC}$ to be in the same range determined for the control group. The variability in the number of MNPE induced by $\mathrm{BC}$ in the evaluated schedule was small and nonsignificant (between 0.6 and 1.6). On the contrary, the administration of $\mathrm{AD}$ revealed a significant increase in all four post-administration points, with the high effects presented at 48 and $72 \mathrm{~h}$. The PE/NE index determined in this protocol is shown in Table 2. No significant modification by BC was observed on the production of PE; however AD significantly reduced the index starting at 72 and $96 \mathrm{~h}$, where a $53 \%$ and $36 \%$ suppression of the erythrocyte production was registered, respectively. AD is a widely used topoisomerase inhibitor antineoplastic agent that is known to cause reactive oxygen species, DNA damage, and apoptosis. ${ }^{8}$ ) Since the chemical has also been reported to induce severe chromosome damage in mice by measuring sister chromatid exchanges (SCE) and micronuclei (MN), it is used as a positive agent in experimental studies. ${ }^{9,10)}$

The results obtained in the assay with three administrations of $\mathrm{BC}$ are presented in Tables 3 and 4 . The first table shows that the level of micronuclei induced by $\mathrm{BC}$ with the two low doses tested along the study was similar to the one detected in the control animals; in contrast, as regards the high dose we found an increase in the rate of MNPE from 48 to $96 \mathrm{~h}$ of the experiment, although without statistical significance. Considering the high level of such a dose, we feel that the obtained result does not imply a genotoxic risk by $\mathrm{BC}$ in our model. AD induced a significant increase in the level of micronuclei with respect to the control value during the four evaluation days. Table 4 shows that, once again, $\mathrm{BC}$ was un-

Table 1. Effect of a Single Administration of Beta-Caryophyllene (BC) on the Rate of Micronucleated Polychromatic Erythrocytes (MNPE) in Mouse

\begin{tabular}{cccccc}
\hline \hline \multirow{2}{*}{$\begin{array}{c}\text { Time } \\
\text { (h) }\end{array}$} & $\begin{array}{c}\text { Corn oil } \\
0.4 \mathrm{ml}\end{array}$ & $\begin{array}{c}\mathrm{BC} \\
20 \mathrm{mg} / \mathrm{kg}\end{array}$ & $\begin{array}{c}\mathrm{BC} \\
200 \mathrm{mg} / \mathrm{kg}\end{array}$ & $\begin{array}{c}\mathrm{BC} \\
2000 \mathrm{mg} / \mathrm{kg}\end{array}$ & $\begin{array}{c}\text { Adryamicin } \\
2.5 \mathrm{mg} / \mathrm{kg}\end{array}$ \\
\cline { 2 - 6 } & & & & & \\
\hline 0 & $1.7 \pm 0.22$ & $1.6 \pm 0.39$ & $1.8 \pm 0.37$ & $1.8 \pm 0.66$ & $2.8 \pm 0.37$ \\
24 & $2.0 \pm 0.31$ & $1.4 \pm 0.39$ & $1.2 \pm 0.48$ & $3.0 \pm 0.31$ & $8.6 \pm 1.02^{*}$ \\
48 & $1.6 \pm 0.26$ & $1.6 \pm 0.24$ & $1.4 \pm 0.51$ & $2.0 \pm 0.70$ & $40.4 \pm 1.77^{*}$ \\
72 & $2.3 \pm 0.26$ & $1.4 \pm 0.6$ & $2.6 \pm 0.68$ & $2.2 \pm 0.58$ & $50.0 \pm 3.18^{*}$ \\
96 & $2.2 \pm 0.37$ & $2.4 \pm 0.68$ & $1.8 \pm 0.48$ & $1.4 \pm 0.39$ & $30.6 \pm 1.86^{*}$
\end{tabular}

The indicated results correspond to the mean \pm S.E.M. of 5 mice per dose. 1000 polychromatic erythrocytes scored per mouse. * Statistically significant difference with respect to the control value ANOVA and Student $t$ tests, $p=0.001$. able to produce a cytotoxic effect in the bone marrow of the treated animals while AD did have this effect at $96 \mathrm{~h}$ of its administration.

Terpenes, and $\mathrm{BC}$ in particular, are promising agents with various useful therapeutic properties; moreover, $\mathrm{BC}$ is a constituent in about $39 \%$ of cosmetic products. ${ }^{4}$ ) Therefore, they deserve to be solidly scrutinized as regards their toxic and genotoxic effects to assure their safe use. Studies on the group have given varying results: for example, in a bacterial assay linalool and $\mathrm{BC}$ showed no mutagenicity but lynalil acetate did; on the other hand, linalool was not antimutagenic whereas BC gave positive results; and finally, the toxicity of linalool and lynalil acetate proved to be stronger than $\mathrm{BC} .^{2)}$ Cytotoxic data about sesquiterpenes in mammalian cells have

Table 2. Effect of a Single Administration of Beta-Caryophyllene (BC) on the Relationship Polychromatic Erythrocytes/Normochromatic Erythrocytes (PE/NE Percent) in Mouse

\begin{tabular}{cccccc}
\hline \hline \multirow{2}{*}{$\begin{array}{c}\text { Time } \\
\text { (h) }\end{array}$} & Corn oil & $\begin{array}{c}\text { BC } \\
20 \mathrm{mg} / \mathrm{kg}\end{array}$ & $\begin{array}{c}\text { BC } \\
200 \mathrm{mg} / \mathrm{kg}\end{array}$ & $\begin{array}{c}\text { BC } \\
2000 \mathrm{mg} / \mathrm{kg}\end{array}$ & $\begin{array}{c}\text { Adryamicin } \\
2.5 \mathrm{mg} / \mathrm{kg}\end{array}$ \\
\cline { 2 - 6 } & & & & & \\
\hline 0 & $2.88 \pm 0.26$ & $2.90 \pm 0.07$ & $2.88 \pm 0.07$ & $2.64 \pm 0.07$ & $3.12 \pm 0.13$ \\
24 & $2.76 \pm 0.06$ & $2.58 \pm 0.08$ & $3.06 \pm 0.22$ & $3.08 \pm 0.09$ & $3.12 \pm 0.11$ \\
48 & $2.83 \pm 0.10$ & $2.60 \pm 0.13$ & $2.74 \pm 0.17$ & $3.22 \pm 0.13$ & $2.50 \pm 0.10$ \\
72 & $2.77 \pm 0.02$ & $2.60 \pm 0.15$ & $2.80 \pm 0.11$ & $3.02 \pm 0.21$ & $0.28 \pm 0.18^{*}$ \\
96 & $2.94 \pm 0.06$ & $2.68 \pm 0.13$ & $2.94 \pm 0.13$ & $3.66 \pm 0.15$ & $0.82 \pm 0.08^{*}$ \\
\hline
\end{tabular}

The indicated results correspond to the mean \pm S.E.M. of 5 mice per dose. 1000 erythrocytes per mouse scored to determine the PE/NE percent. * Statistically significant difference with respect to the control value ANOVA and Student $t$ tests, $p=0.001$.

Table 3. Effect of Three Administrations of Beta-Caryophyllene (BC) on the Rate of Micronucleated Polychromatic Erythrocytes (MNPE) in Mouse

\begin{tabular}{cccccc}
\hline \hline & \multicolumn{5}{c}{ MNPE percent $(\bar{X} \pm$ S.E.M. $)$} \\
Time \\
\cline { 2 - 6 }$(\mathrm{h})$ & Corn oil & $\begin{array}{c}\text { BC } \\
20 \mathrm{mg} / \mathrm{kg}\end{array}$ & $\begin{array}{c}\mathrm{BC} \\
200 \mathrm{mg} / \mathrm{kg}\end{array}$ & $\begin{array}{c}\text { BC } \\
2000 \mathrm{mg} / \mathrm{kg}\end{array}$ & $\begin{array}{c}\text { Adryamicin } \\
2.5 \mathrm{mg} / \mathrm{kg}\end{array}$ \\
\hline 0 & $2.4 \pm 0.24$ & $2.4 \pm 0.68$ & $2.2 \pm 0.37$ & $2.2 \pm 0.58$ & $2.5 \pm 0.25$ \\
24 & $2.2 \pm 0.48$ & $2.2 \pm 0.37$ & $1.4 \pm 0.39$ & $2.2 \pm 0.56$ & $18.0 \pm 0.81$ \\
48 & $2.4 \pm 0.39$ & $0.8 \pm 0.48$ & $1.8 \pm 0.37$ & $4.2 \pm 0.42$ & $24.0 \pm 0.96^{*}$ \\
72 & $2.2 \pm 0.48$ & $2.6 \pm 0.74$ & $1.6 \pm 0.51$ & $4.5 \pm 0.77$ & $23.0 \pm 1.15^{*}$ \\
96 & $3.0 \pm 0.54$ & $2.2 \pm 0.37$ & $1.8 \pm 0.37$ & $4.7 \pm 0.31$ & $11.2 \pm 1.05^{*}$
\end{tabular}

The indicated results correspond to the mean \pm S.E.M. of 5 mice per dose. 1000 poly chromatic erythrocytes scored per mouse. $*$ Statistically significant difference with respect to the control value ANOVA and Student $t$ tests, $p=0.01$.

Table 4. Effect of Three Administrations of Beta-Caryophyllene (BC) on the Relationship Polychromatic Erythrocytes/Normochromatic Erythrocytes (PE/NE Percent) in Mouse

\begin{tabular}{cccccc}
\hline \hline & \multicolumn{5}{c}{ PE/NE percent $(\bar{X} \pm$ S.E.M. $)$} \\
\cline { 2 - 6 } Time \\
\cline { 2 - 6 } & Corn oil & $\begin{array}{c}\text { BC } \\
20 \mathrm{mg} / \mathrm{kg}\end{array}$ & $\begin{array}{c}\text { BC } \\
200 \mathrm{mg} / \mathrm{kg}\end{array}$ & $\begin{array}{c}\text { BC } \\
2000 \mathrm{mg} / \mathrm{kg}\end{array}$ & $\begin{array}{c}\text { Adryamicin } \\
2.5 \mathrm{mg} / \mathrm{kg}\end{array}$ \\
\hline 0 & $2.46 \pm 0.18$ & $2.80 \pm 0.15$ & $3.14 \pm 0.10$ & $2.78 \pm 0.11$ & $2.80 \pm 0.09$ \\
24 & $2.58 \pm 0.11$ & $2.84 \pm 0.18$ & $3.26 \pm 0.17$ & $2.32 \pm 0.20$ & $2.50 \pm 0.13$ \\
48 & $2.78 \pm 0.13$ & $2.92 \pm 0.16$ & $3.02 \pm 0.19$ & $2.80 \pm 0.20$ & $2.67 \pm 0.08$ \\
72 & $2.76 \pm 0.13$ & $3.08 \pm 0.20$ & $3.04 \pm 0.13$ & $2.85 \pm 0.18$ & $2.35 \pm 0.05$ \\
96 & $2.66 \pm 0.06$ & $3.16 \pm 0.18$ & $2.88 \pm 0.09$ & $3.12 \pm 0.22$ & $1.77 \pm 0.11^{*}$
\end{tabular}

The indicated results correspond to the mean \pm S.E.M. of 5 mice per dose. 1000 erythrocytes per mouse scored to determine the PE/NE percent. * Statistically significant difference with respect to the control value ANOVA and Student $t$ tests, $p=0.01$. 
also varied; for example, in cultivated cells a stronger effect of alpha humulene is generally shown, in comparison with that of alpha or gamma caryophyllene, BC being the less toxic of these agents. ${ }^{1)}$ In regard to genotoxic studies in mammalian cells, no previous studies on the BC potential have been made. However, contrary to our negative results and those of Di Soto et al. in bacteria, ${ }^{2)}$ other sesquiterpenes have given positive results: this is the case of the compound lactone lynchnopholide that was reported as a chromosome damaging agent in in vitro and in vivo assays, and was shown to be cytotoxic in vitro ${ }^{11)}$; another such case is that of parthenin which presented a strong capacity to induce micronuclei in vivo. ${ }^{12}$ These divergent results suggest the relevance of the chemical structure in the biological effect of sesquiterpenes, and indicate as well the importance of using various test models to reach a valid conclusion. Concerning this last point, Morales et al. compared the effect of 11 sesquiterpenoids in the Ames and the V79/HGPRT tests, and they found congruence in only four of them. ${ }^{13)}$

During its metabolism, BC can originate hydroperoxides as primary oxidation products; these, however, are rapidly converted to caryophyllene oxide, which is a low reactive and more stable product. ${ }^{4)}$ This process, together with efficient DNA repair and detoxification by mouse, may be involved in the absence of a significant number of micronuclei induced by the chemical. In summary, the results obtained in the present experiment suggest using $\mathrm{BC}$ safely for industrial and therapeutic purposes, although this should be confirmed in other test models.

\section{REFERENCES}

1) Legault J., Pichette A., J. Pharm. Pharmacol., 59, 1643-1647 (2007).

2) Di Sotto A., Evandri M. G., Mazzanti G., Mutat. Res., 653, 130-133 (2008).

3) Politeo O., Jukić M., Milos M., Croat. Chem. Acta, 79, 545-552 (2006).

4) Sköld M., Karlberg A. T., Matura M., Börge A., Food Chem. Toxicol., 44, 538-545 (2006).

5) da Silva S. L., Figueiredo P. M., Yano T., Eur. J. Pharmacol., 576, $180-188$ (2007).

6) Lorke D., Arch. Toxicol., 54, 275-287 (1983).

7) Hayashi M., Sofuni T., Ishidate M., Mutat. Res., 120, 241-247 (1983).

8) Mizutani H., Tada-Oikawa S., Hiraku Y., Kojima M., Kawanishi S., Life Sci., 76, 1439-1453 (2005).

9) Bommu P., Nanjan C. M., Joghee N. M., Nataraj S. M., Bhojraj S., Nat. Med., 62, 149-154 (2008).

10) Paniagua-Pérez R., Madrigal-Bujaidar E., Reyes-Cadena S., AlvarezGonzález I., Sánchez-Chapul L., Pérez-Gallaga J., Hernández N., Flores-Mondragón G., Velasco O., Arch. Toxicol., 82, 615-622 (2008).

11) Canalle R., Burim R. V., Callegari Lopes J. L., Takahashi C. S., Cancer Detect. Prev., 25, 93-101 (2001).

12) Ramos A., Rivero R., Visozo A., Piloto J., Garcia S., Mutat. Res., 514, $19-27$ (2002).

13) Morales P., Andersson M., Lewan L., Sterner O., Mutat. Res., 268, 315-321 (1992). 